\title{
Paraneoplastic hypocalcemia in a patient whit gastric cancer accompanied by osteoblastic metastasis
}

\author{
Abdolali Shahrasbi ${ }^{1}$, Kosar Heydarzadeh ${ }^{2}$, Sepideh Fahimi ${ }^{3}$, Nazila Abbasi fard $^{4}$ \\ ${ }^{I}$ Assistant Professor, Department of Medical Oncology, Bu Ali Hospital, Tehran Medical Science Branch, Islamic Azad \\ University of Tehran, Tehran, Iran \\ ${ }^{2}$ Medical Student, Tehran Medical Sciences Branch, Islamic Azad University of Tehran, Tehran, Iran
}

\begin{abstract}
Paraneoplastic syndromes are generally defined as clinical disorder associated with malignant diseases, and hypocalcemia as paraneoplastic manifestation associated with cancer is a rare condition. In patient with bone metastatic cancer, osteolytic lesions are more common than osteoblastic ones which give rise to hypercalcemia and hypocalcemia is a rare condition in this group of patients. We described a 56-year-old male with the history of gastric cancer and gastrectomy and chemotherapy since one year ego who came to clinic with complaints of nausea, vomiting and paresthesia of extremities which after diagnostic work up, paraneoplastic hypocalcemia was diagnosed. Hypocalcemia following bone metastasis is not a common phenomenon, but in patients with the history of gastric cancer and neurological symptoms (paresthesia, ...) evaluation of serum calcium value is recommended.

Keywords: Hypocalcemia, Gastric cancer, Bone metastasis.

Cited as: Shahrasbi A, Heydarzadeh K, Fahimi S, Abbasi fard N. Paraneoplastic hypocalcemia in a patient whit gastric cancer accompanied by osteoblastic metastasis. Medical Science Journal of Islamic Azad University, Tehran Medical Branch 2020; 30(2): 221-225.
\end{abstract}

Correspondence to: Abdolali Shahrasbi

Tel: +989121309264

E-mail: a_shahra@hotmail.com

ORCID ID: 0000-0003-2629-1179

Received: 1 Jun 2019; Accepted: 14 Sep 2019 
مجله علوم يزشكى دانشَاه آزاد اسلامى

Case

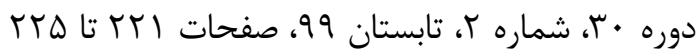

Report

\section{بررسى هييو كلسمى یار انئو يلاستيك در يك بيمار مبتلا به سرطان معده همراه با متاستاز به استخوان}

\section{عبدالعلى شهراسبى', كوثر حيدرزاده؟، سييده فهيمى ؟، نازيلا عباسى فرد؟}

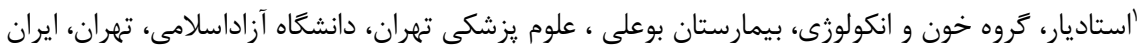

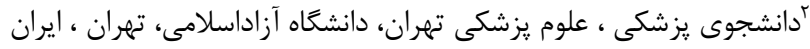

جكيده

سندرم بارانئوبلاستيك /ز تظاهرات بالينى مرتبط با سرطان است و هييوكلسمى به عنوان تظاهر بارانئويالاستيك در سرطانها بسيار نادر

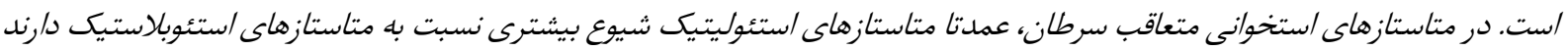

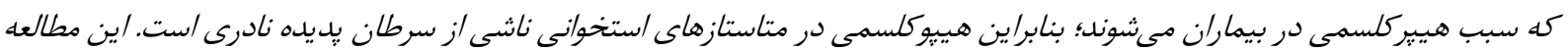

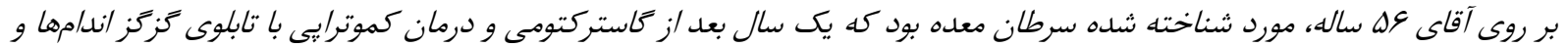

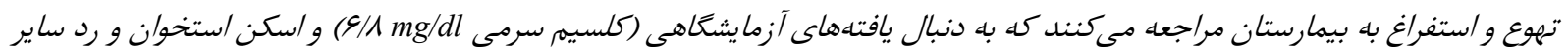

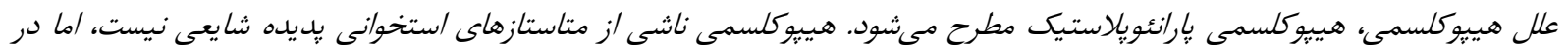

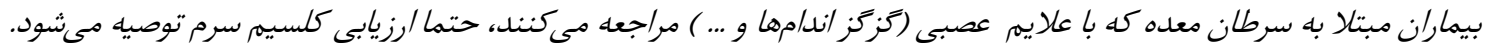
وازكان كليدى: هييوكلسمى، سرطان معده، متاستاز /ستخوانى.

هييوكلســمى نوعى اختلال متابوليكى نادر اســت كه گمكاه در مقلمه

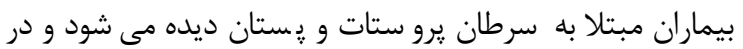

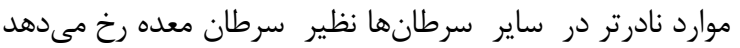

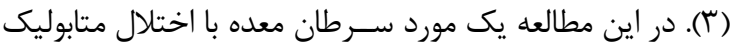

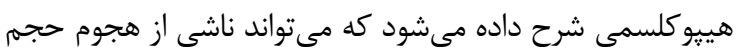

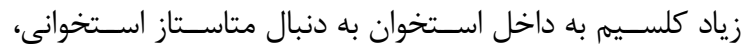

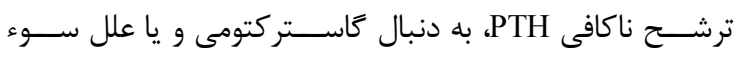

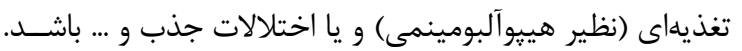

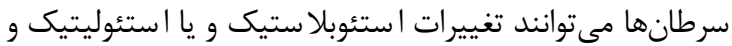

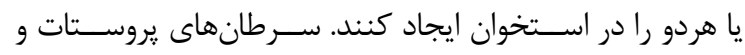

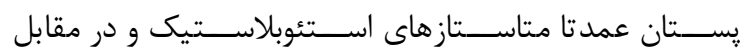
سرطانهاى نواحى ديخر عمدتا متا ستازهاى ا ستئوليتيك ايجاد 
كلوكونات و كلســى تريول خوراكى روزانه قرار كرفت و روزانه

سطح سرمى كلسيم و فسفر קك شد (نمودار ().

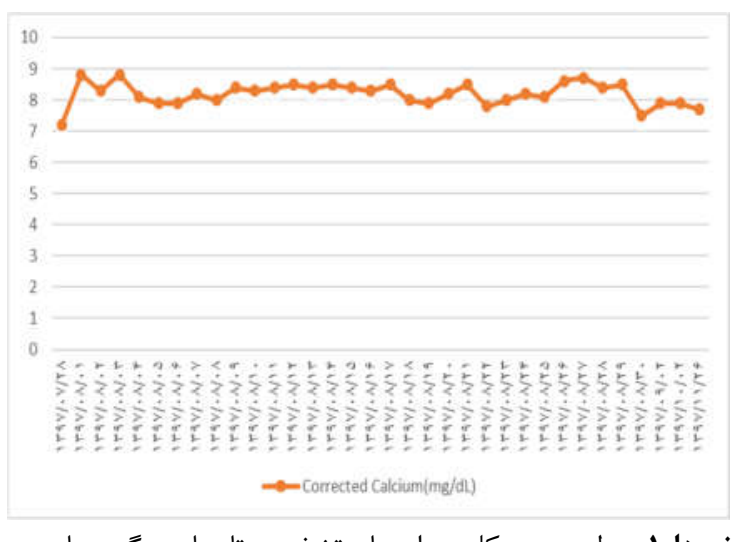

نمودار ا. سطح سرمى كلسيم از زمان تشخيص تا زمان مرك بيمار

\begin{tabular}{|c|c|c|}
\hline محدوده & ميانه & \\
\hline $1 \cdot-90$ & TAV & PTH(mg/dl) \\
\hline$r / \Delta-\Delta / \Delta$ & $r / 4$ & $\mathrm{P}(\mathrm{mg} / \mathrm{dl})$ \\
\hline $1 / 9-T / r$ & $r$ & $\operatorname{Mg}(\mathrm{mg} / \mathrm{dl})$ \\
\hline$r \cdot-q$. & $r \mu / q$ & 1,25-(OH)Vit.D(Pg/dl) \\
\hline $\mid r F-1 F \Delta$ & $1 \pi q / r$ & $\mathrm{Na}(\mathrm{mEq} / \mathrm{L})$ \\
\hline$r / \Psi-\Delta / r$ & $r / \Lambda r$ & $\mathrm{~K}(\mathrm{mEq} / \mathrm{L})$ \\
\hline$r / \Delta-\Delta / 1$ & $r / V$ & $\operatorname{Albumin}(\mathrm{g} / \mathrm{dl})$ \\
\hline$\cdot / 0-1 / \Delta$ & ו & Creatinin(mg/dl) \\
\hline
\end{tabular}

\begin{tabular}{|c|c|c|}
\hline محدوده & ميانه & \\
\hline$f \cdots-11 \cdots$ & rv. . & W.B.C $(/)$ \\
\hline$\varphi / r \cdot-4$ & $r / l V$ & R.B.C $(10 * 6 /)$ \\
\hline$|r-| V / \Delta \cdot$ & $11 / \cdot$ & $\mathrm{Hb}(\mathrm{g} / \mathrm{dl})$ \\
\hline$r \Lambda-\Delta r$ & 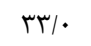 & HCT(\%) \\
\hline$\Lambda \cdot-1 \cdot$. & V৭/l & $\operatorname{MCV}(\mathrm{fl})$ \\
\hline ra-rr & $r g / 4$ & $\mathrm{MCH}(\mathrm{pg})$ \\
\hline ML & 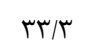 & $\mathrm{MCHC}(\mathrm{g} / \mathrm{dl})$ \\
\hline 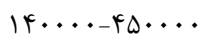 & 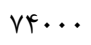 & Platelet(/) \\
\hline $1 \cdot \mid \Delta-1 F / \Delta$ & $19 / 1$ & RDW(\%) \\
\hline
\end{tabular}

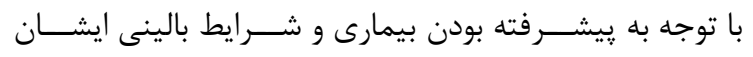
performance status2 (ECOG scale)

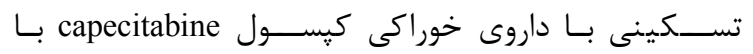

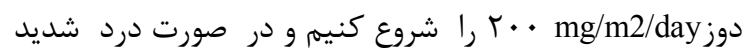

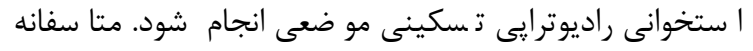

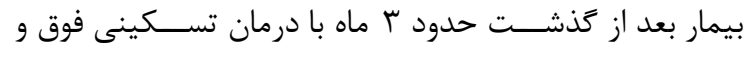

مى كنند. سرطان معده به ندرت متا ستازهاى ا ستئوبلا ستيك

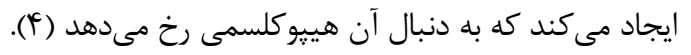

\section{كَزارش مورد}

بيمار آقاى \&ه ســالهاى از حدود يك ســال قبل به دنبال درد

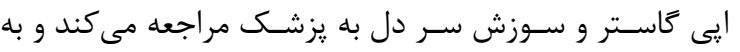

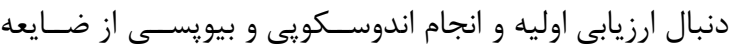

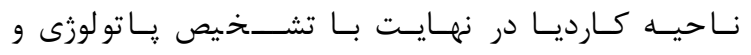
poorly differentiated adenocarcinoma ايمونوهيستوشيمى به كلينيك انكولوزى ارجاع شـــــ بيمار تحت يروتكل درمانى (مطابق تريال (magic) تحت سه نوبت كموترايى Neoadjuvant

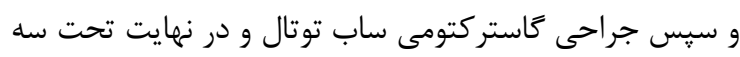

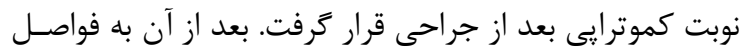

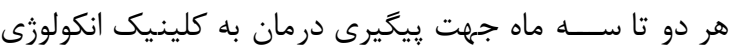

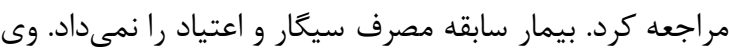

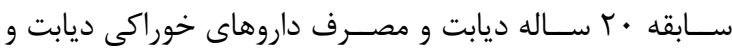

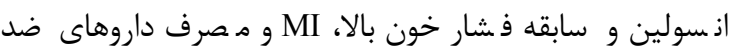

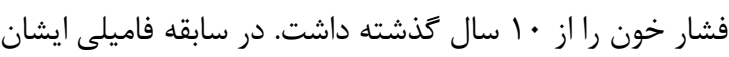

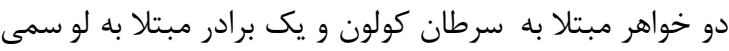
حاد (ALL) وجود داشت

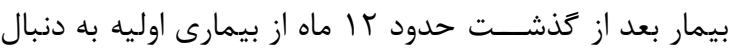

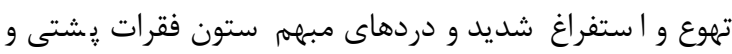

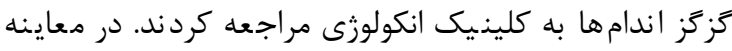

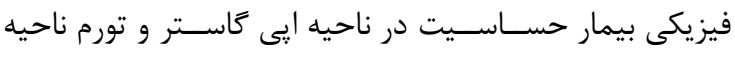

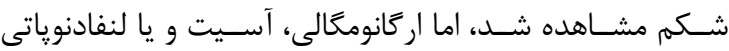

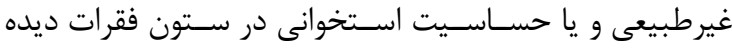

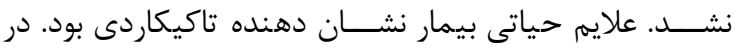

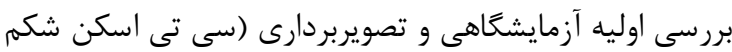

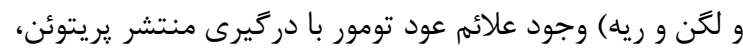

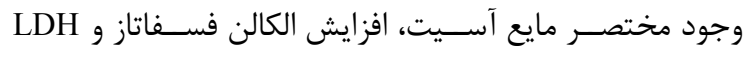

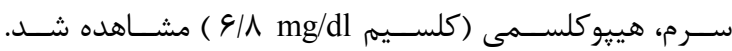

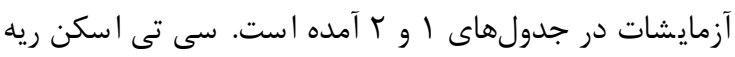

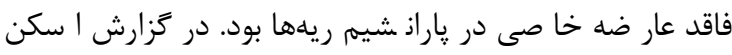

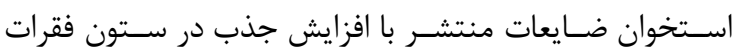

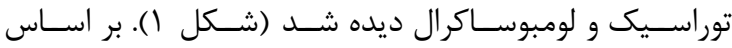

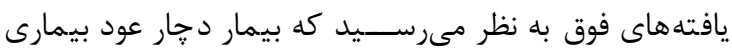

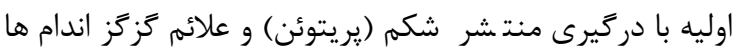

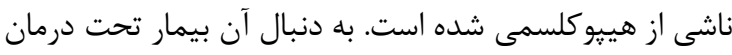

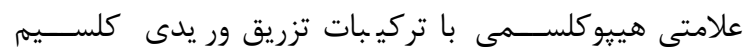


ســـوح غير طبيعى فســفر و منيزيم، ميزان ناكافى هورمون

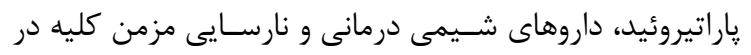

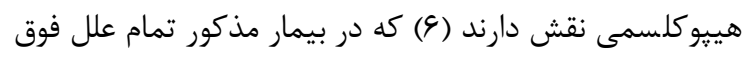

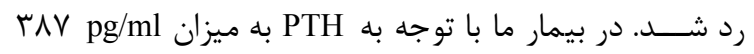

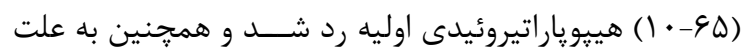

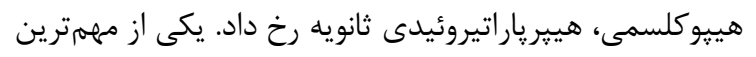

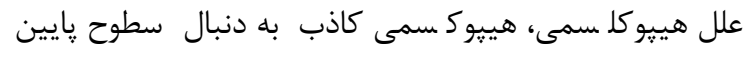

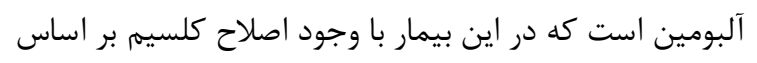

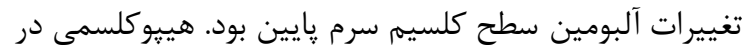

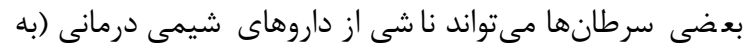

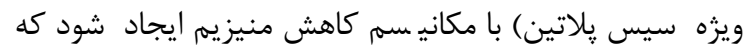

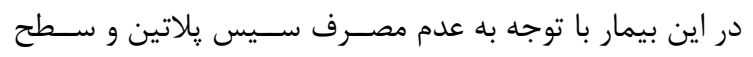

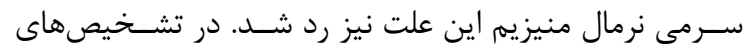

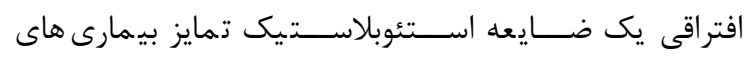
متاستاتيك استخوانى از بيمارى هاى متابوليك استخوان بسيار

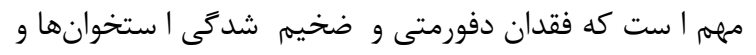

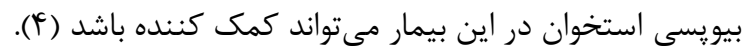

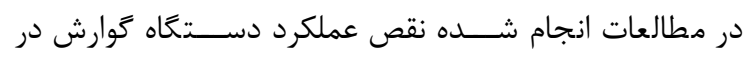
سرطان معده صرف نظر از گا ستركتومى ممكن ا ست يكى از مكانيزمهاى هييو كلسمى باشد كه در مطالعه ما سطح فريتين

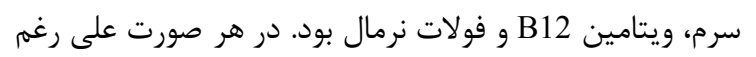

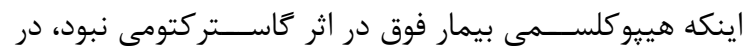

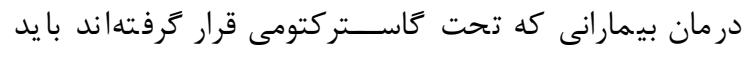

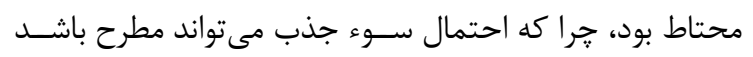

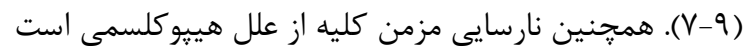

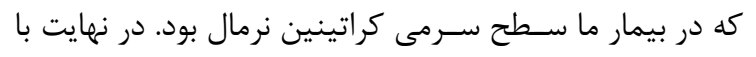

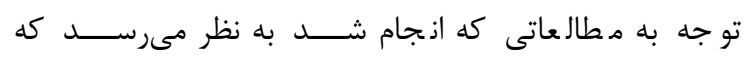
مكانيسمهاى مولكولى متفاوتى ناشى از سلولهاى سرطانى، در

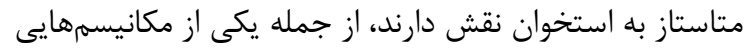

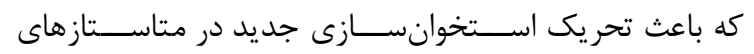

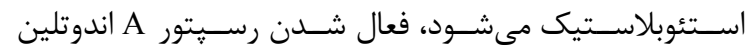

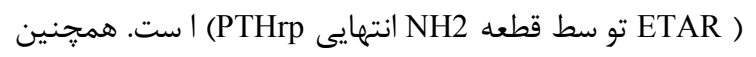
فاكتورهاى رشــد ترشــح شــده PDGF IGF و آدرنومدولين

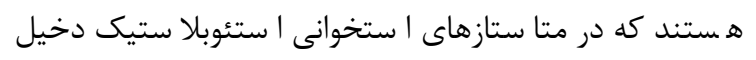

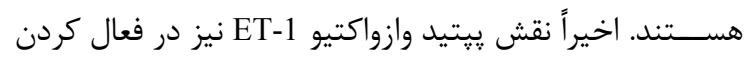

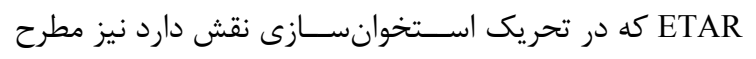

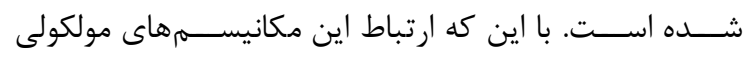

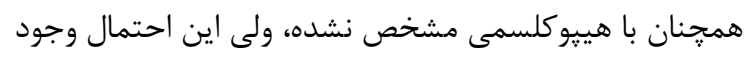

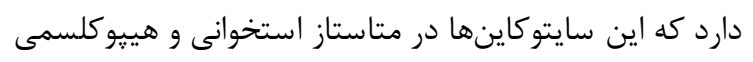

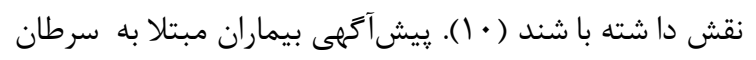

كنترل نســبى هييو كلســمى در نهايت با يِيشـرفت بيمارى و دركيرى منتشــر يريتوئن و ضـايعات متاسـتاتيك ريوى در در فاز نارسايى تنفسى فوت كردند.

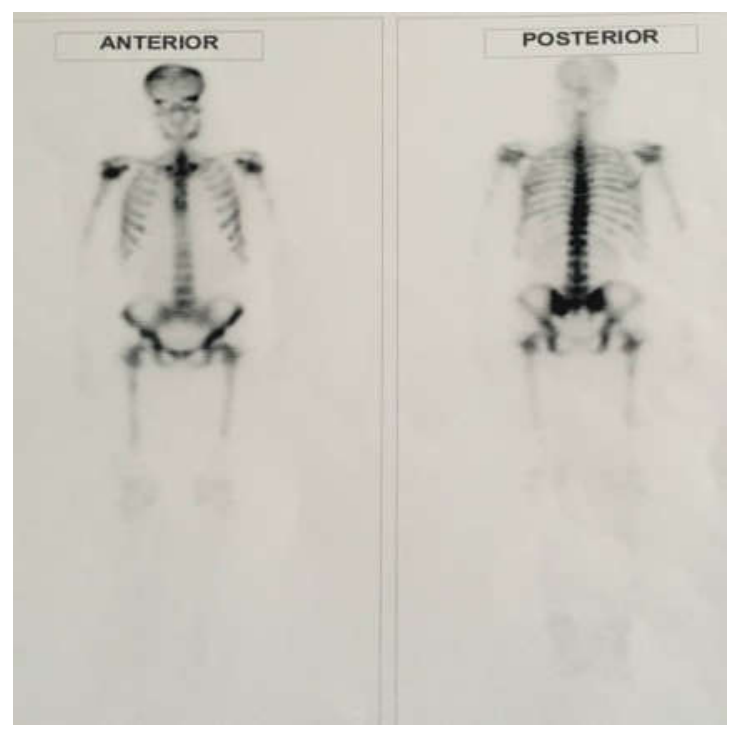

شكل ا. اسكن استخوان با استفاده از تكنسيوم 199م كه افزايش جذب در اسكلت استخوانى را نشان مىدهد.

بحث متاستازهاى استخوانى عمدتاً در سرطانهاى يرو ستات، ريه و

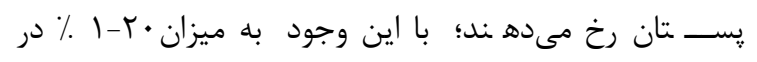
سرطانهاى معده نيز شاهد متاستازهاى استخوانى هستيم. اين

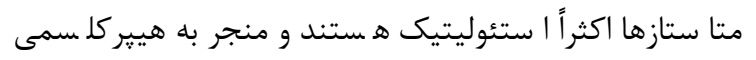
مىشـوند، البته درصــ كمترى مى توانند اسـتئوبلاسـتيكى نيز با شند. در متا ستازهاى ا ستئوبلا ستيك سطح آلكالن فسفاتاز

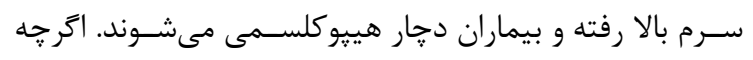

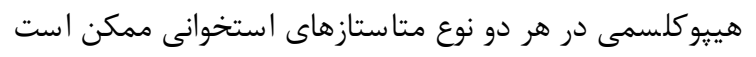

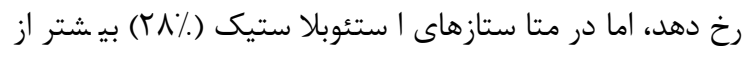

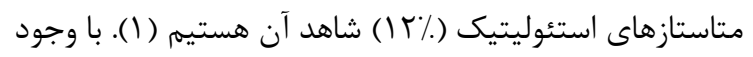

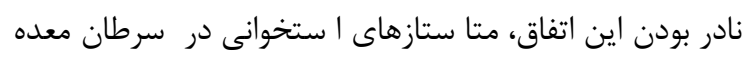

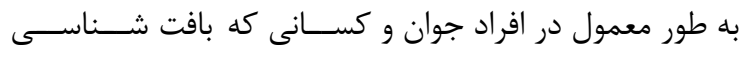

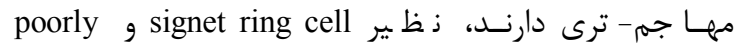
differentiated gastric cancer

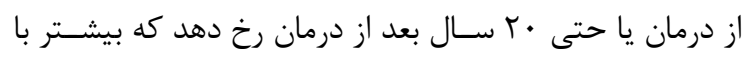

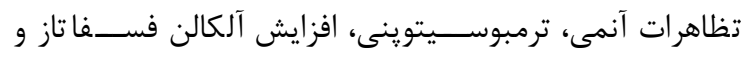

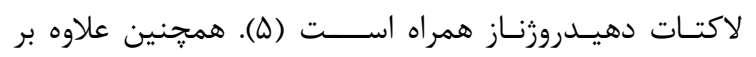

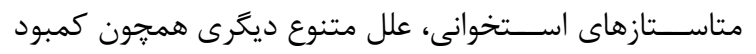

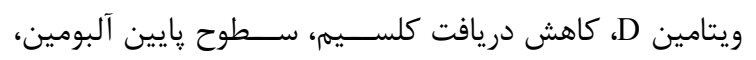




$$
\begin{aligned}
& \text { شايعتر است، اما در بيماران مبتلا به سرطان كه با علائم عصبى } \\
& \text { معده همراه با متاســتاز اســتخوانى بســيار بد اسـت و عمدتاً }
\end{aligned}
$$

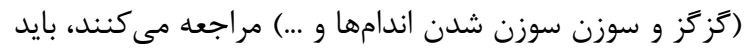

$$
\begin{aligned}
& \text { بيماران در فاصله جهار ماه از تشخيص فوت مى كنند (11). } \\
& \text { هييو كلسمى و اندازه گيرى سطح سرمى آن را مدنظر قرار داد. } \\
& \text { هييوكلسمى ناشى از متاستازهاى استخوانى يديده شايعى مئى }
\end{aligned}
$$

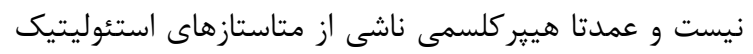

\section{REFERENCES}

1. Okazaki J, Muguruma N, Kitamura S, Kimura T, Okamoto K, Miyamoto H, et al. Paraneoplastic hypocalcemia developed in gastric cancer accompanied by osteoblastic metastasis. Intern Med 2017; 56: 1345-1349.

2.Dimitriadis GK, Angelousi A, Weickert MO, Randeva HS, Kaltsas G, Grossman A. Paraneoplastic endocrine syndromes. Endocr Relat Cancer 2017;24: R173-R190.

3.Raskin P, McClain CJ, Medsger TA. Hypocalcemia associated with metastatic bone disease: a retrospective study. Arch Intern Med 1973;132: 539-543.

4.Chung YS, Choi TY, Ha CY, Kim HM, Lee KJ, Park CH, et al. An unusual case of osteoblastic metastasis from gastric. Yonsei Med J 2002;43: 377-380.

5.Fonocho E, Aydin N, Reddy S, Misra S. Recurrent gastric cancer metastasizing to the bone marrow: A case report of a rare presentation. Int J Surg Case Rep 2017;37: 165-168.

6.Diniotis B, Sternberg E, Shakuntala S, Chiha M, Khosla P. Hypocalcemia in malignancy-unexpected but common. Cureus 2015; 7:12-18.

7.Iizumi S, Shimoi T, Nishikawa T, Kitano A, Sasada S, Shimomura A, et al. Prolonged hypocalcemia following a single dose of denosumab for diffuse bone metastasis of gastric cancer after total gastrectomy. Intern Med 2017; 56: 2879-2882, 2017.

8.Radigan AE. Post-gastrectomy: managing the nutrition fallout. Pract Gastroenterol 2004;28: 63-79.

9.Szentirmai M, Constantinou C, Rainey JM, Loewenstein JE. Hypocalcemia due to avid calcium uptake by osteoblastic metastases of prostate cancer. West J Med 2001; 163: R577.

10.Guise TA, Mohammad, KS, Clines G, Stebbins EG, Wong DH, Higgins LS, et al. Basic mechanisms responsible for osteolytic and osteoblastic bone metastases. Clin Cancer Res 2006;12: 6213s-6216s.

11.Kim HS, Yi SY, Jun HJ, Lee J, Park JO, Park YS, et al. Clinical outcome of gastric cancer patients with bone marrow metastases. Oncology 2011;73: 192-197. 